\title{
Wie entstehen wissenschaftliche Subjekte? Zum professoralen Ethos akademischer Lebenspraxis
}

\author{
Julian Hamann, Forum Internationale Wissenschaft, Universität Bonn
}

\begin{abstract}
Der Beitrag rückt Praktiken der akademischen Subjektivierung in den Fokus. Das Genre akademischer Nachrufe erlaubt eine differenzierte Betrachtung der Praktiken, die wissenschaftliche Sprecherfiguren diskursiv konstituieren. Aufbauend auf eine qualitative Analyse von 216 Nachrufen rekonstruiert der Beitrag, welche Kriterien an legitime Wissenschaftssubjekte angelegt werden. Es wird gezeigt, dass Fleiß, Hingabe, sowie wissenschaftliche und institutionelle Verdienste im Zentrum der Subjektwerdung stehen, während sozialstrukturelle Determinanten wie soziale Herkunft oder Geschlecht systematisch ausgeblendet werden. Indem Nachrufe auf diese Weise wissenschaftliche Subjekte konstituieren, reproduzieren sie ein allgemeines, professionell-professorales Ethos akademischer Lebenspraxis. Sie können dann als Konsekrationsinstanzen verstanden werden, die dreifach wirken: erstens als feierliche Ehrung vergangener Leben, zweitens als Auszeichnung derjenigen Autorinnen, die diese Ehrung vornehmen können, und drittens als Instanz, die die Regeln des Feldes als Maßstab für alle Agenten reifiziert. Der Beitrag behandelt damit einen (innerwissenschaftlichen) Mechanismus, der für die Entstehung (auch außerwissenschaftlich wirksamer) wissenschaftlicher Sprecher zentral ist.
\end{abstract}

\section{Einleitung: Wie entstehen wissenschaftliche Subjekte? ${ }^{1}$}

Zu den zentralen Fragen hinsichtlich der machtvollen Beziehung zwischen der Wissenschaft und anderen Gesellschaftsbereichen gehört die Frage, wer als autorisierter akademischer Sprecher auftreten kann. Wer sind die Produzenten und Repräsentantinnen ${ }^{2}$ wissenschaftlichen Wissens? Wie wird man zu jemandem, der in der Position ist, wissenschaftliche Wahrheit zu sprechen? Was sind und wie entstehen wissenschaftliche Subjekte? In diesem Beitrag untersuche ich Nachrufe als Instanzen, die bestimmte Subjekte konstituieren und konsekrieren und damit sichtbar machen, welche Erwartungen an akademische Lebensläufe gestellt werden. Auch die noch lebenden Subjekte werden durch Nachrufe geweiht, weil ihre Karrieren und Werke nach

\footnotetext{
${ }^{1}$ Für hilfreiche Hinweise zu einer früheren Version dieses Textes danke ich Vincent Gengnagel, Alexander Hirschfeld, Jens Maeße und Christian Schmidt-Wellenburg.

${ }^{2}$ Im Sinne der einfacheren Lesbarkeit wechselt der Text zwischen maskulinem und femininem Genus ab. Soweit nicht anders vermerkt sind immer beide Genera gemeint, auch wenn dies nicht immer die Realität widerspiegelt: Im hier zugrundeliegenden Korpus wurden 93 Prozent der Nachrufe von männlichen Wissenschaftlern geschrieben und 95 Prozent handeln von männlichen Wissenschaftlern.
} 
eben jenen Regeln Bedeutung erlangt haben, die im Nachruf evoziert werden. Die in Nachrufen aufscheinende Wahrheit, der sich damit alle Beteiligten verschreiben, (v-)erklärt Erfolg mit subjektivem Talent und Fleiß sowie mit wissenschaftlichen und institutionellen Meriten, während Merkmale wie soziale Herkunft oder Geschlecht invisibilisiert werden.

Die Wissenschaftsforschung interessiert sich schon seit geraumer Zeit für die Praktiken, die wissenschaftliches Wissen hervorbringen, und für die institutionellen Strukturen, von denen dieses Wissen gerahmt wird. Die Relevanz einer Perspektive auf die Träger wissenschaftlichen Wissens liegt auf der Hand, wenn man an den beachtlichen gesellschaftlichen Einfluss denkt, den beispielsweise Ärztinnen in Form von Gutachten, Ökonomen durch Beratungsleistungen, Sozialwissenschaftler mit vielfältigen Expertisen, Ingenieurinnen in der Technikfolgenabschätzung und geisteswissenschaftliche Interventionen im Feuilleton haben. Wenn wissenschaftliche Wahrheiten generiert werden, ist ihre Wirksamkeit ganz wesentlich abhängig davon, dass sie von einer bestimmten Position aus geäußert und einer konkreten Sprecherin zugeordnet werden können. Den zentralen Stellenwert subjektivierter Sprecherpositionen verdeutlichen auch in der Wissenschaft selbst so unterschiedliche Phänomene wie die soziale Struktur von Schulen und Communities, Denominationen von Lehrstühlen sowie Instanzen wie Zitationen und die Idee der Autorschaft. Wissenschaftliches Wissen wird also situativ hervorgebracht und es ist institutionell gerahmt, es wird aber auch von spezifischen Subjektpositionen aus produziert, reproduziert, getragen und vertreten. Bei diesen Subjekten handelt es sich um wahrgenommene Entitäten, die adressierbar und zurechenbar sind, denen bestimmte Fähigkeiten, Verantwortungen oder Eigenschaften zugemessen werden und die wissenschaftlich wahre Aussagen machen können (Etzemüller 2013; Reckwitz 2006).

Wissenschaftliche Subjekte können ihre Expertisen, Claims, Beratungen und Interventionen wirkungsvoll in verschiedensten gesellschaftlichen Kontexten platzieren (vgl. die Beiträge von Hirschfeld und Gengnagel, Maeße, sowie Schmidt-Wellenburg in diesem Band). Die Subjektform „Wissenschaftler“ wird damit zu einer „Funktionsposition“ (Etzemüller 2013: 188), die wissenschaftlich Sagbares und Relevantes markieren kann. Eine wichtige Quelle, aus der diese Aussagen und Positionen ihre gesellschaftliche Autorität schöpfen, liegt jedoch im wissenschaftlichen Feld selbst. Dort werden Trägerinnen wissenschaftlichen Wissens in einer Vielzahl von Arenen konstituiert: wissenschaftliche Texte bringen Autoren diskursiv in Erscheinung (Angermuller 2014; Lamont 1987), Curricula Vitae strukturieren Lebensläufe als kohärente, lineare Biographien (Dietz et al. 2000; Hahn 1988), Gremien und Netzwerke richten Persönlichkeiten und Habitus zu (Beaufaÿs 2003; Engler 2001), bürokratische Verfahren und Prozesse 
bringen institutionelle Positionen wie „Doktorand von...“ oder „Professorin für...“ hervor (Musselin 2009; Zimmermann 2004; vgl. auch den Beitrag von Angermuller in diesem Band).

So zentral diese Szenarien der Subjektkonstitution sind, so schwierig ist es genau zu benennen, nach welchen Kriterien und Regeln wissenschaftliche Subjekte hervorgebracht werden. Welche Maßstäbe und Ansprüche, welche Normen und welches Ethos liegen zugrunde, wenn wissenschaftliche Subjekte konstruiert, geformt, diszipliniert, institutionalisiert werden? Ohne größeren Aufwand ließen sich die Kriterienkataloge und Richtlinien identifizieren, die in Gutachten für Forschungsprojekte (Lamont 2009) und Zeitschriftenartikel (Hirschauer 2010) oder in Empfehlungsschreiben (Tsay et al. 2003) formuliert sind. Die Entstehung von ganzheitlich gedachten Personen, die wissenschaftliche Wahrheit sprechen und akademische Autorität ausüben können, bleibt dagegen weitgehend im Dunkeln. Es ist ein empirisches Desiderat, wie kontingente Stationen individueller Lebensläufe überführt werden in den logischen und stringenten Verlauf einer Biographie, die einer Person zugeschrieben wird und ein sinnhaftes wissenschaftliches Subjekt überhaupt erst sichtbar macht (zu dieser „biographischen Illusion“ vgl. Bourdieu 1998a).

Mein Beitrag folgt der Frage nach der Subjektwerdung von Wissenschaftlerinnen. Die Kernthese lautet, dass akademische Nachrufe allgemeinere Regeln der Konstitution wissenschaftlicher Subjekte offenbaren. Als Konsekrationsinstanzen verleihen Nachrufe Verstorbenen die letzte Weihe, weil sie sie als holistisches wissenschaftliches Subjekt adressieren. Konsekriert werden dabei auch die Autorinnen der Nachrufe, sie erscheinen als Priesterinnen, die diesen Akt im Namen der Gemeinschaft vollziehen können. Dabei explizieren sie die Kriterien für legitime Subjekte und reifizieren diese als Maßstab für alle Agenten des Feldes. Als Arenen symbolischer Herrschaft geben Nachrufe also den Blick frei auf bestimmte Erwartungen an Subjekte, während sie andere Regeln und Kriterien verschleiern oder tabuisieren. Ich gehe also davon aus, dass Nachrufe ein Panorama professionell-professoraler Werte anrufen, dem nicht nur bereits verstorbene Subjekte genügen müssen. Auch für noch lebende Wissenschaftlerinnen, „Etablierte“ wie „Außenseiter“, besteht der Druck, ihre akademische Lebensweise den in Nachrufen aufscheinenden Kriterien legitimer Forschungsbiographien anzugleichen. Nachrufen sind also nicht zuletzt ein interessanter Untersuchungsgegenstand, weil die symbolische Konstitution von Wissenschaftssubjekten ineinander greift mit sedimentierten sozialen Stratifikationen (siehe dazu auch Maeße und Hamann 2016).

Diese Argumentation entwickelnd skizziere ich im Folgenden zunächst einige Forschungsstränge, die sich mit der Hervorbringung und Positionierung wissenschaftlicher Subjekte be- 
schäftigen (2.). Daraus können dann methodologische Besonderheiten wissenschaftlicher Subjektivierungspraktiken abgeleitet werden (3.1), aufgrund derer sich spezifische textförmige Genres der Subjektivierung (3.2) und bestimmte Methoden (3.3) besonders für eine empirische Untersuchung eignen. Im Hauptteil meines Beitrags rekonstruiere ich Kriterien und Merkmale der Subjektwerdung aus dem empirischen Material. Die Struktur des zugrunde liegenden Samples erlaubt es, dabei universelle Kriterien wie „Fleiß“ und „Hingabe“ (4.1) zu unterscheiden von Zuschreibungen, die länderspezifisch oder zeitabhängig variieren („Talent“" in Deutschland, „Internationalität“ sowie eine generelle Quantifizierung von Leistungsmerkmalen im Zeitverlauf; 4.2). Ebenso relevant sind schließlich Merkmale wie soziale Herkunft oder Geschlecht, die im Rahmen der Subjektkonstitution gerade nicht thematisiert werden (4.3). Der letzte Abschnitt diskutiert diese empirischen Befunde. Ich werde argumentieren, dass es sich bei Nachrufen um Konsekrationsinstanzen handelt, die dreifach wirken: erstens als feierliche Ehrung vergangener Leben, zweitens als Auszeichnung derjenigen Autorinnen, die diese Ehrung vornehmen können, und drittens als Instanz, die die Regeln des Feldes als Maßstab für alle Agenten reifiziert (5.).

\section{Wissenschaftliche Normen, Positionierungen und Subjekte}

Die Frage, wie die gesellschaftlich adressierbare (und adressierende) Figur des „Wissenschaftlers" entsteht, von der aus wissenschaftliche Wahrheit gesprochen werden kann, überspannt drei Forschungsfelder. Theoretische und gesellschaftsanalytische Studien beschäftigen sich mit Subjektivierungsdynamiken und Subjektbegriffen. Diese Überlegungen werden empirisch fruchtbar gemacht von Arbeiten zu wissenschaftlichen Positionierungen. Sie zeigen, wie Wissenschaftlerinnen welche Positionen besetzen. Dabei spielen schließlich akademische Werte und Normen eine Rolle, die erstens situativ immer wieder neu ausgehandelt werden müssen und deswegen zweitens kontingent und kontextabhängig sind.

Ein knapper Einblick in die modernere Subjektivierungsforschung referiert am besten selbst auf drei wissenschaftliche Subjekte: „Judith Butler“, „Michel Foucault“ und „Louis Althusser“. Die Subjektfigur „Louis Althusser“ (1977) steht dafür, Subjekte als eine ideologische Anrufung zu konzipieren. Sie sind damit durch Ideologien wie zum Beispiel Religion und ihre oftmals ritualisierten, institutionalisierten Anrufungspraktiken konstituiert. Das Subjekt „Michel Foucault“ (1976) betrachtet das Subjekt als einen Machteffekt, über den Individuen in MachtWissen-Komplexen geformt und diszipliniert werden - zum Beispiel als „Kriminelle“ oder „Soldaten“. In späteren Arbeiten verschiebt sich der Fokus dann auf feiner verästelte Regierungstechniken, die von Subjekten selbst ausgeführt werden (Foucault 2010). In Anlehnung an 
die diskursive Subjektposition „Foucault“ geht auch „Judith Butler“ (1997) von der performativen Wirkmächtigkeit von Sprache aus. Wenn Sprechakte Handlungsqualität besitzen, so ihre Annahme, dann lassen spezifische Sprachformen spezifische Subjektformen in Erscheinung treten. Im Gegensatz zu strukturalistischen Perspektiven kann Butler die Subjektwerdung auch als dynamischen, iterativen Prozess beschreiben, der Bedeutungsverschiebungen, Sinnüberschüsse und Widerstände produzieren kann (vgl. Reckwitz 2008).

Ein Desiderat der auf diesen und anderen Pfeilern aufbauenden Subjektivierungsforschung bleibt, dass neben theoretischen und gesellschaftsanalytischen Arbeiten, die beispielsweise unternehmerische, hybride, dezentrierte und Beratungssubjekte ausrufen (Bröckling 2007; del Pierco et al. 2014; Reckwitz 2006; Traue 2010), nur wenige Studien existieren, die Dynamiken und Praktiken der Subjektivierung empirisch rekonstruieren (dazu auch Alkemeyer 2013). Bis hier können wir demnach ein umfassendes Theorieangebot bilanzieren, auf das auch mein Beitrag aufbauen wird. Die Frage, wie aus Individuen und ihren mehr oder weniger brüchigen und kontingenten Lebensläufen empirisch ein sozial lesbares und intelligibles Subjekt wird, ist jedoch noch nicht systematisch adressiert worden. Empirische Zugänge werden immerhin angedeutet bei Martin Kohli (1981), der die Fragestellung jedoch vor allem soziologiegeschichtlich einordnet und wiederum in erster Linie methodologisch beleuchtet, bei Loïc D. Wacquant (2000), der für (auto-)biographische Darstellungen von Wissenschaftlern an Fallbeispielen aufzeigt, welche Zensureffekte wirksam werden, in den Beiträgen von Thomas Etzemüller (2013, 2015), der ebenfalls schlaglichtartig die performative Logik wissenschaftlicher Subjekte rekonstruiert. Auch diese Autoren identifizieren die systematische empirische Erforschung der Subjektivierung von Wissenschaftlerinnen jedoch als Desiderat.

Instruktiv sind vor diesem Hintergrund Studien zu Positionierungen in der Wissenschaft. Sie gehören nicht zur Subjektivierungsforschung im engeren Sinne, stehen aber durchaus in ihrer poststrukturalistischen Tradition. Ihr Fokus liegt auf der Vielfalt von Praktiken und Dynamiken, die beeinflussen oder strukturieren, wie Wissenschaftlerinnen welche Positionen einnehmen und anderen zuschreiben. So ist auf die strukturellen Bedingungen von Positionierungen aufmerksam gemacht worden, indem gezeigt wurde, wie intellektuelle, kulturelle, institutionelle und soziale Faktoren die Legitimität einer Sprecherposition beeinflussen. Diese Arbeiten zeigen, wie wissenschaftliche Subjekte und Sprecherpositionen - beispielsweise ,ökonomischer Experte“ oder „Jacques Derrida“ - konstituiert werden. Sie heben dabei die Bedeutung der jeweiligen Kontextbedingungen hervor, zum Beispiel in der deutschen Geschichtswissenschaft der 1940er bis 1960er Jahre (Etzemüller 2013), im akademischen Paris der 1960er Jahre (Lamont 1987) oder in der globalen politischen Ökonomie (Maeße 2015a). Die Herstellung von 
Subjektpositionen ist auch als praktisches Problem beschrieben worden. Wissenschaftlerinnen müssen ihre Position und ihren Platz immer wieder neu finden, definieren und verteidigen, sie sind darüber hinaus mit den Fremdpositionierungen ihrer Peers konfrontiert. Dies ist der Fall in ineinander verschränkten Wissen-Macht-Komplexen (Angermuller 2013; 2014, siehe auch den Beitrag von Angermuller in diesem Band; vgl. auch Baert 2012) und auch bei der Grenzarbeit zwischen Wissensfeldern (Abbott 1995; Lamont und Molnár 2002). Weiterhin wurde untersucht, welchen Einfluss intellektuelle Selbstkonzepte und -narrative auf die Selbstkonstitution wissenschaftlicher Subjekte haben (Engler 2001; Gross 2002; Lamont et al. 2000). Schließlich betonen Arbeiten in diesem Forschungsstrang die Bedeutung von Macht und Konflikten. Sie heben symbolische Kämpfe hervor, in denen Wissenschaftler aufgrund ihrer Ausstattung mit verschiedenen Kapitalsorten prä-reflexive Positionierungsstrategien verfolgen (Bourdieu 1992), und beschreiben in Habitusanalysen den praktischen Sinn von Zuschreibungs- und Positionierungspraktiken (Beaufaÿs 2003).

Eng verbunden mit Arbeiten zu wissenschaftlichen Positionierungen ist die Frage, auf welche Werte und Normen die Positionszuschreibungen rekurrieren, um legitime und von ihren Peers anerkannte Subjekte zu konstituieren. Studien zu diesem Thema erinnern daran, dass die Positionen, Grenzen und Praktiken der akademischen Welt vielfältig, ambivalent und fluide sind (Becher und Trowler 2001). Die meisten Studien über akademische Normen und Werte betonen erstens, dass diese situativ und kontextabhängig ausgehandelt werden. Zweitens erstrecken sie sich über ein breites Spektrum von Werten, das sich von „Originalität“ (Guetzkow et al. 2004) und „Intellekt“ (Tsay et al. 2003) über „Charisma“ (Gustin 1973) bis „Exzellenz“ (Lamont 2009; vgl. Peter 2014) erstreckt.

Die drei skizzierten Forschungsstränge bieten wichtige Einsichten zur Frage, wie eine gesellschaftlich adressierbare (und adressierende) Figur des „Wissenschaftler“ entsteht, von der aus wissenschaftliche Wahrheit gesprochen werden kann. Gleichzeitig macht der Forschungsstand jedoch auch die methodologischen und methodischen Probleme sichtbar, die sich mit einer Perspektive auf Subjektivierungen von ,Wissenschaftlern“ verbinden. Aus der Diskussion dieser Probleme heraus werde ich im nächsten Abschnitt begründen, warum sich Nachrufe in besonderer Weise als empirische Daten für Subjektivierungen eignen.

\section{Die empirische Erforschung der Subjektivierung von ,Wissenschaftlern`}

Der Forschungsstand hat deutlich gemacht, dass die Erforschung der Subjektwerdung von Wissenschaftlerinnen insbesondere zwei methodologische und methodische Besonderheiten mit sich bringt. Im Folgenden will ich nicht nur diese Eigentümlichkeiten diskutieren (3.1), sondern 
auch die vielfältigen Möglichkeiten aufzeigen, der Subjektwerdung von Wissenschaftlerlinnen dennoch empirisch auf die Spur zu kommen (3.2). Am Ende des Abschnitts stelle ich den methodischen Ansatz meines eigenen Beitrags vor (3.3). Zunächst jedoch zu den bereits im Forschungsstand deutlich gewordenen Schwierigkeiten.

\subsection{Methodologische Besonderheiten von Subjektivierungen}

Eine erste Besonderheit der Subjektivierung von, Wissenschaftlern` ist, dass Subjekte im wissenschaftlichen Feld oft gerade durch ihre Negation gekennzeichnet sind. Wissenschaftssubjekte werden nicht einfach beschrieben, sondern häufig auch beschwiegen (zur wissenschaftlichen Selbstbeschweigung vgl. Etzemüller 2013; Kohli 1981). In der Regel wird Subjektives und Individuelles von den Zensurmechanismen des wissenschaftlichen Diskurses sanktioniert. Nicht umsonst schreibt Max Weber (1922: 533) der Wissenschaftsforschung in ihr Taufbuch, dass „,Persönlichkeit“ auf wissenschaftlichem Gebiet“" nur der habe, „der rein der Sache dient“،. Robert K. Merton (1973: 276) abstrahiert dann sogar die Norm selbst von der Person und beschreibt das programmatische Desinteresse am Subjektiven als grundlegendes institutionelles Element „of science itself“. Ganz praktisch zeigt sich dieser Zensurmechanismus dann beispielsweise in der Vermeidung des ,Ichs“ und der Verwendung selbstreferentieller Passivkonstruktionen in wissenschaftlichen Aufsätzen (Gusfield 1976). Stimmt man Pierre Bourdieu (2004) zu, dass eben jene Interessenlosigkeit genau das Interesse der Wissenschaft ist, dann wird die Beschweigung des Subjekts zum Teil der Illusio, und ihre Rekonstruktion zu einem wichtigen Beitrag zum Verständnis des wissenschaftlichen Feldes und seiner Agenten.

Eine zweite Schwierigkeit bringt der Untersuchungsgegenstand mit sich, weil es sich um hochgradig personalisierte und teilweise informelle Praktiken und Dynamiken handelt. Die empirische Erforschung von Subjektivierungen hat sich daher in der Regel damit beholfen, entsprechende Daten durch teilnehmende Beobachtung oder Interviews zu erheben. In beiden Fällen besteht aber die Gefahr einer Responsivität der Datenerhebung für Fragestellungen, Erwartungen oder auch nur die Anwesenheit der Forscherin, was beispielsweise zu einem (Antwort-)Verhalten nach sozialer Erwünschtheit führen kann (Häder 2010). Teilnehmende Beobachtungen stehen zusätzlich vor der Herausforderung, im akademischen Alltag verstreute und jeweils unterschiedlich situierte Praktiken zu allgemeineren Subjektivierungsdynamiken aggregieren zu müssen. Für Interviews kann außerdem der Einwand erhoben werden, dass es sich hierbei selbst um einen „Biographiegeneratoren“ (Hahn 1988:93) handelt, der demzufolge einen neuen Kontext für die Hervorbringung ganz eigener Subjektivierungspraktiken schafft (vgl. auch Bernhard 2014). 


\subsection{Empirische Daten von Subjektivierungen}

Bis hierhin wurde der (gesellschafts-)theoretische Forschungsstand zu Subjektivierungen sowie die Literatur zu akademischen Subjektpositionen und Wertzuschreibungen skizziert und die sich daraus ergebenden Erkenntnisse, aber auch die resultierenden Schwierigkeiten des Untersuchungsgegenstands bilanziert. Das ist der Hintergrund, vor dem nun textförmige Daten zur Subjektwerdung von ,Wissenschaftlern“ diskutiert werden können. Trotz der methodologischen Sperrigkeit von Subjektivierungspraktiken als Untersuchungsgegenstand erlaubt eine Reihe von Genres einen textbasierten Zugriff, der die Schwierigkeiten von Interviews und teilnehmender Beobachtung umgehen kann. Prominente Orte der textförmigen Konstitution von Wissenschaftssubjekten sind neben Nachrufen auch Curricula Vitae (CVs) (siehe dazu Bonzi 1992; Dietz et al. 2000) und die literarische Form der Biographie (siehe dazu Shortland und Yeo 1996; Söderqvist 2011). In allen Fällen handelt es sich um Biographiegeneratoren, um Arrangements also, die auf die biographieförmige Abbildung von Subjektfiguren ausgerichtet sind.

Nachrufe adressieren im Gegensatz zu CVs und Biographien wissenschaftliche Schulen, Gemeinschaften, oder ein gesamtes Fach. Ein verlässlicher Indikator hierfür ist, dass sie in wissenschaftlichen Zeitschriften und in den Veröffentlichungen von Fachverbänden publiziert werden. Im Unterschied zu anderen Genres sind Nachrufe außerdem auf die feierliche Erinnerung akademischer Lebensleistungen ausgerichtet. Das ehrliche Urteil ist hier nur selten gefragt, so dass es auch nicht überraschen kann, wenn negative Aspekte wie Kritik, Brüche oder Ungereimtheiten im Lebenslauf tendenziell ausgeblendet oder umdefiniert werden. Umso aufschlussreicher ist es, diese Darstellungsregeln als Ausdruck der Illusio des Feldes zu interpretieren. Die enge Beziehung, die Verstorbene und Autoren in Nachrufen in der Regel verbindet, hat eine wichtige Funktion: Sie führt dazu, dass Autorinnen von Nachrufen als legitime Sprecher erscheinen, die den Verstorbenen im Namen der Schule, der Gemeinschaft oder der Disziplin in angemessener Form präsentieren und klassifizieren dürfen, deren Urteil aber auch von diesen Kollektiven gelesen und damit überwacht wird. ${ }^{3}$

Bereits diese knappe Skizze macht deutlich, dass Nachrufe in ganz bestimmten Kontexten und zu konkreten Zwecken produziert und rezipiert werden. Diese Situierung in jeweils spezifischen sozialen Konfigurationen muss in der Analyse reflektiert werden. Deutlich geworden ist auch, dass es in Nachrufen mitnichten nur um das Leben der Verstorbenen geht. Vielmehr handelt es sich bei dem Genre auch um Arenen, in denen sich ein Forschungsfeld über sich

\footnotetext{
${ }^{3}$ Nicht umsonst hebt Bourdieu (1992: 379) hervor, dass Klassifikations- und Konsekrationsakte in Nachrufen von einem „dazu ordnungsgemäß beauftragten Sprecher” vorgenommen werden, gleichzeitig aber ,immer das Produkt kollektiver Arbeit" sind.
} 
selbst verständigt, in denen zulässige Praktiken der Subjektivierung aktualisiert und reifiziert werden, und in denen Bedingungen der Mitgliedschaft zu einem akademischen Kollektiv verhandelt werden. Diese Inhalte werden vor allem durch Narrative intelligibel gemacht, die Subjektfiguren festschreiben und (re-)produzieren (zum Konzept und der identitären Bedeutung des Narrativs vgl. Bamberg 2010; Koschorke 2012). Aus methodologischer Sicht besteht das Potential von Nachrufen also nicht zuletzt darin, dass sie Subjekte nicht unsichtbar machen, sondern eben gerade explizieren. Die Subjektivierung läuft hier vor allem diskursiv ab (und zum Beispiel weniger praktisch-materiell, vgl. Mialet 1999). Sie ist charakterisiert durch ein relationales Verhältnis von Selbstpositionierung (der Autorin), Fremdpositionierung (des Verstorbenen) und einem mitlesenden Publikum, das beide Positionierungen bezeugen und gegebenenfalls sanktionieren kann. Auf diese Weise dokumentieren Nachrufe in besonders deutlicher Weise, dass Subjektivierungsdynamiken keine monadischen Abläufe sind, die ein Individuum mit sich selbst ausmacht. In das Genre ist bereits explizit ein Beziehungsgeflecht von Schülern, Lehrern, Weggefährten, Kollegen, Förderern und anderen Subjekten eingebaut.

Bisherige Studien zu Nachrufen haben diese Stärken des Genres verdeutlicht. In Zeitungen veröffentlichte Nachrufe können als Beiträge zu einem kollektiven Gedächtnis verstanden werden. Sozialstrukturelle Erhebungen zeigen dann an, wen eine Gesellschaft in Nachrufen erinnert (Fowler 2005a; siehe auch Fowler 2005b, 2007; Fowler und Bielsa 2007) und welche Rolle beispielsweise Geschlechtsdifferenzen spielen (Eid 2002; Rodler et al. 2001). Nachrufe in Zeitungen erlauben zweitens in stärker konstruktivistischer Perspektive einen Blick auf die Repräsentation und Symbolisierung von Identitäten (Bonsu 2007; Long 1987) oder von bestimmten Erwartungen und Vorurteilen (Bytheway und Johnson 1996). An diese Arbeiten knüpfen Studien an, die sich speziell mit akademischen Nachrufen auseinandersetzen. Dazu gehören Gispens (1989) Studien zur Ingenieurelite, die sich eher auf sozialstrukturelle Faktoren konzentrieren, Tights (2008) stärker konstruktivistisch ausgerichtete Perspektive darauf, inwiefern Nachrufe den starken Einfluss der US-amerikanischen Wissenschaft und der Universitäten Oxford und Cambridge widerspiegeln, sowie weitere Studien über Eigenheiten der Disziplin Soziologie (Hamann 2015; für einen sprachwissenschaftlichen Zugang vgl. Kresta 1996), über genealogische Wertungspraktiken (Echterhölter 2012; Hamann und Zimmer 2016), informelle Quellen intellektueller Reputation (Macfarlane und Chan 2014) und über biographische Narrative und Positionierungspraktiken (Hamann 2016).

Zusammenfassend ist festzuhalten: Nachrufe erlauben einen Zugriff auf die relationale Konstitution von ansonsten oft beschwiegenen Wissenschaftssubjekten. Sie konsekrieren die Verstorbenen, legitimieren die Autorinnen, die diesen letzten Akt der Subjektivierung im Namen 
der Gemeinschaft ausführen können, und reifizieren feldspezifische Werte für alle Agenten des Feldes. Dem analytischen Blick erschließen sich so jene symbolischen Feldstrukturen, die Subjekte und ihre Biographien erst ermöglichen und hervorbringen.

\subsection{Untersuchungsdesign und Methode}

Bis hierhin habe ich argumentiert, dass sich aus Nachrufen typische, charakteristische Subjektivierungspraktiken rekonstruieren lassen, mit denen Wissenschaftler als Subjekte hervorgebracht werden. Dieses Vorhaben verfolge ich anhand von über 841 Nachrufen, die in zentralen akademischen Journals und den Publikationen von Fachverbänden veröffentlicht wurden. Aus dieser Population habe ich ein Sample von 216 Nachrufen ausgewählt. Stratifiziertes Sampling (Patton 2002) hat eine hinreichende Varianz zwischen den Fällen gewährleistet und ermöglicht, drei theoretisch begründete Vergleichsdimensionen in das Sample einzuführen (Kelle und Kluge 2010; Strauss und Corbin 1990):

- Die Nachrufe im Sample sind in Deutschland, den USA oder UK veröffentlicht. Sie bilden damit zentrale Hochschulsysteme ab, deren Governance im Zeitverlauf in unterschiedlichem Ausmaß zwischen nationalstaatlicher und neoliberaler Ausrichtung changiert (Ben-David 1977; Moore et al. 2011).

- Durch Kontrolle der veröffentlichenden Fachzeitschrift, der Autorin und des Verstorbenen können die Nachrufe im Sample der Physik, der Geschichtswissenschaft oder der Soziologie zugeordnet werden. Dies bildet ein Spektrum von Natur- über Sozial- bis zu den Geisteswissenschaften ab (Kagan 2009).

- Die Nachrufe im Sample sind in den 1960er, den 1980er oder den 2000er Jahren veröffentlicht. Diese Phasen erfassen wichtige Trends im Hochschulsektor wie beispielsweise Neoliberalisierung (Giroux 2014) oder Internationalisierung (Huang et al. 2014).

Das Sample wurde gerichtet und disproportional zusammengestellt (Emmel 2013), so dass in jeder Subpopulation eine ausreichende Zahl von Fällen vorhanden ist (Tabelle 1). ${ }^{4}$

Tabelle 1: Verteilung von Nachrufen auf Vergleichsdimensionen

\begin{tabular}{ll|l|l|l|l} 
& $1960-1970$ & $1980-1990$ & $2000-2010$ & Total \\
\hline \multirow{2}{}{} & Physik & 8 & 8 & 8 & 72 \\
\hline
\end{tabular}

\footnotetext{
${ }^{4}$ Der begrenzende Faktor war hier die britische Soziologie, für die aufgrund ihrer besonderen Entwicklung und insbesondere ihrer späten Institutionalisierung nur eine begrenzte Zahl von Nachrufen auf britische Soziologen aus den 1960er Jahren auffindbar war (siehe dazu Halsey 2004).
} 


\begin{tabular}{|c|c|c|c|c|c|}
\hline & Soziologie & 8 & 8 & 8 & \\
\hline \multirow{3}{*}{ 光 } & Physik & 8 & 8 & 8 & \multirow{3}{*}{72} \\
\hline & Geschichtswissenschaft & 8 & 8 & 8 & \\
\hline & Soziologie & 8 & 8 & 8 & \\
\hline \multirow{3}{*}{ 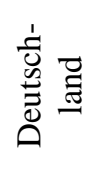 } & Physik & 8 & 8 & 8 & \multirow{3}{*}{72} \\
\hline & Geschichtswissenschaft & 8 & 8 & 8 & \\
\hline & Soziologie & 8 & 8 & 8 & \\
\hline Total & & 72 & 72 & 72 & 216 \\
\hline
\end{tabular}

Insgesamt liegen dem Sample 59 Publikationsorte zugrunde, und zwar sowohl akademische Journals (z.B. British Journal of Sociology für Soziologie in UK, Journal of Modern History für die US-Geschichtswissenschaft) als auch Publika von Fachvertretungen (z.B. Footnotes für die US-Soziologie, Physikalische Blätter/Physik Journal für die deutsche Physik).

Nachrufe aus dem theoretischen Sample wurden in einer ersten Phase relativ offen kodiert. Diese eng am empirischen Material orientierte, inhaltsgeleitete Kategorisierung von Daten identifiziert verbreitete oder charakteristische Themen und Praktiken. In dieser Runde konnten verschiedene Kodes zur Konstitution akademischer Subjekte gebildet werden. Beispielsweise hat sich gezeigt, dass ,hohe Anerkennung bei internationalen Peers' oder ,Forschungsaufenthalt im Ausland‘ typische Zuschreibungen sind. In einer zweiten Phase, dem axialen Kodieren, wurden diese Kodes verknüpft oder verfeinert, bis ein Punkt der theoretischen Sättigung erreicht war, an dem keine neuen Unterthemen oder Beziehungen mehr rekonstruiert werden konnten. Diese Vorgehensweise konnte Kriterien und Eigenschaften von Wissenschaftssubjekten identifizieren, die zumindest für die im Sample enthaltenen Nachrufe als typisch und charakteristisch gelten können (zum Kodieren im Sinne der Grounded Theory vgl. Strauss und Corbin 1990).

\section{Wie werden wissenschaftliche Subjekte konstituiert?}

Auf Grundlage des beschriebenen Samples lassen sich die Kriterien und Maßstäbe rekonstruieren, entlang derer Subjekte in Nachrufen charakteristischerweise konstituiert und als Idealbild typisiert werden. Meine Analyse lenkt den Blick zunächst auf allgemeine Aspekte, die über alle Vergleichsdimensionen hinweg typisch sind (4.1). Weiterhin lassen sich Subjektivierungspraktiken identifizieren, die variieren und anhand derer sich vor allem nationale und temporäre Spezifika aufzeigen lassen (4.2). Abschließend kann unter Rückgriff auf den Forschungsstand zu akademischen Karrieren gefragt werden, welche Rolle sozialstrukturelle Determinanten für Subjektivierungspraktiken spielen (4.3). 
4.1 Wissenschaftliche und institutionelle Verdienste, Fleiß und Hingabe

In allen Disziplinen, Phasen und Ländern des Samples werden wissenschaftlichen Subjekten verschiedene Verdienste zugeschrieben. ${ }^{5}$ Vor allem wissenschaftliche Verdienste stellen ein zentrales Maß dar, das im Nachruf an das Subjekt angelegt wird. So kann akademische Relevanz signalisiert werden, wenn ein Soziologe „die neuere Weber-Rezeption in Deutschland wesentlich angeregt hat" (Lepsius 1986, S. 415), Beiträge zur methodischen Entwicklung eines Faches werden angedeutet wenn hervorgehoben wird, dass ein Forscher „developed the photographic method for the Zenith telescope thereby cutting in half the probable errors of the latitude determinations as compared with the visual method used up to that time." (van Biesbroeck 1961, S. 276) Ein Verdienst um die Fortentwicklung des empirischen Kenntnisstandes mag für einen Historiker darin bestehen ,to have mapped out the intricate pattern of French rural society.“ (Cobb 1960, S. 61) Die Zuschreibung wissenschaftlicher Verdienste, aufteilbar in theoretische, empirische oder methodische Erkenntnisse, spielt demnach eine hervorgehobene Rolle bei der Konstitution von Subjekten.

Neben wissenschaftlichen Meriten ist auch die Zuschreibung institutioneller Verdienste charakteristisch. Institutionelle Relevanz mag beispielsweise darin bestehen, dass von einem Forscher die Methodensektion der Deutschen Gesellschaft für Soziologie (DGS) gegründet, später auch der Vorsitz der DGS übernommen und „,noch viele andere Ämter ausgefüllt“ (Rehberg 2003, S. 821) wurden. Für andere wird aus Posten als ,associate provost“ und „,vice president“ ein „talent for administration“ (Koshiba 2003, S. 82) abgeleitet. Wie kann das Verhältnis der Zuschreibungen wissenschaftlicher und institutioneller Verdienste beschrieben werden? Bei der Anrufung wissenschaftlicher Subjekte konkurrieren institutionelle Verdienste nicht mit wissenschaftlichen Verdiensten, genauso wenig erscheinen andersherum administrative Meriten als Zeichen für mittelmäßige Forschung. Und doch deutet die zentrale Platzierung und relative Häufigkeit der jeweiligen Zuschreibungen an, dass wissenschaftliche Verdienste für die Konstruktion legitimer akademischer Subjekte eine wichtigere Rolle spielen.

Weder für wissenschaftliche noch für institutionelle Verdienste lassen sich im Sample bedeutende Unterschiede zwischen Disziplinen, Ländern oder Zeiträumen ausmachen. Es handelt sich hier also um Maßstäbe, die Wissenschaftssubjekte in allen Kontexten zugeschrieben wurden. Auffallend ist, dass die zugeschriebenen Verdienste den Kapitalsorten entsprechen, ent-

\footnotetext{
${ }^{5}$ Mit diesen Zuschreibungen von Verdiensten oder Meriten vollziehen sich Positionierungen, die ich an anderer Stelle beschrieben habe (Hamann 2016).
} 
lang derer sich nach Bourdieu (1992) akademische Felder aufspannen. Dass akademisches Kapital für die Abbildung legitimer Subjekte eine wichtigere Rolle spielt als institutionelles beziehungsweise weltliches Kapital, mag mit der von feldanalytischen Studien immer wieder diagnostizierten Illusio einer Autonomie des Feldes zusammenhängen, nach der die legitimsten Beiträge noch immer diejenigen sind, die zum Fortschritt ,reiner Erkenntnis ' geleistet werden (Bourdieu 1992; Gengnagel und Hamann 2014; Hamann 2014; Maeße 2015b).

Als wissenschaftliche und institutionelle Verdienste definierte Leistungen werden in Nachrufen auf spezifische Weise erklärt und plausibilisiert. Ein im Sample universelles, über Zeit, Disziplinen und Länder hinweg mobilisiertes Narrativ hierfür ist das der vollkommenen Hingabe. Fast jeder Forscher „devoted himself“ (Outhwaite 1993, S. 387) für sein Fach, die Feststellung, dass „no man gave more - or more effectively - of himself to the discipline” (Stryker 1968, S. 60) gilt als Auszeichnung. Ein ,[r] astlos tätig[es,] [...] ganz der Wirtschaftsgeschichte gewidmetes Leben“ (Aubin 1967, S. 572) erscheint ebenso als ehrenwert wie ein „life in which almost sixty years were devoted to the advancement of astronomy“ (Kopal 1972, S. 265). Charakterliche Zuschreibungen, die eine solche Hingabe und Aufopferungsfähigkeit unterstreichen, heben den Fleiß und die Arbeitsfreude der Subjekte hervor: Ein Verstorbener mag dem Fach „,seine fast bis zum Schluss unermüdliche Arbeitskraft zur Verfügung“"(Conze 1982, S. 301) gestellt haben oder durch eine „,nie müde werdende[n] Leistungsbereitschaft“ (Münch 1987, S. 626) ausgezeichnet gewesen sein.

Eine wichtige Nuancierung der Kriterien des Fleißes und der Hingabe ist, dass hier nicht eifrige und emsige Subjekte beschrieben werden. Als Zuschreibung im Sample haben Fleiß und Hingabe in der Regel nichts Kleingeistiges oder Einfältiges, sondern markieren, dass Wissenschaftler ihre Arbeit aufgrund einer tief verankerten Getriebenheit geradezu genießen. Subjekte zeichnen sich daher aus durch „devotion and enthusiasm“ (Hauser 1961, S. 365), ein Physiker „seemed to enjoy” (van Biesbroeck 1961, S. 277) Überstunden am Teleskop, während ein anderer ,zu den begeisterten Wissenschaftlern [gehörte], von denen die Kollegen auch nach dem dritten Lebensjahrzehnt Gedankenreichtum und sprühenden Arbeitswillen erwarten.“ (Brüche 1970, S. 365)

Die Anrufung von Wissenschaftssubjekten zielt also darauf ab, dass sie sich ihre Verdienste erarbeitet haben und dass erfolgreiche akademische Karrieren auf diese Weise verdient wurden. Hierbei handelt es sich um eine Legitimation im Sinne einer meritokratischen Ideologie (vgl. Hadjar 2008; McNamee und Miller 2004), die nicht nur die Rolle von Kontingenzen und Zu- 
fällen für akademische Lebensläufe ausblendet, sondern auch die Bedeutung, die andere Determinanten wie Geschlecht, familiärer Status oder soziale Herkunft haben (vgl. dazu Abschnitt 4.3). Mit Bourdieu (1998b, 2001) erkennen wir hier eine Ausübung symbolischer Herrschaft, die Erfolg über individuelle Merkmale erklärt, strukturelle Bedingungen invisibilisiert und damit einen Eigenbeitrag der Beherrschten zu ihrer Beherrschung darstellt. Konsekriert werden daher nicht nur die Verstorbenen, sondern auch die Autoren, weil sie diese Wahrheit des Feldes reifizieren und damit auch ihre eigene Position im Feld durch den Verweis auf besondere Leistungs- und Leidensfähigkeit rationalisieren. Gleichzeitig wird mit der Betonung der Hingabe an die akademische Sache ein Anspruch formuliert, der eine dezidiert exkludierende Wirkung haben kann (Beaufaÿs 2015; Paulitz et al. 2015): Das wissenschaftliche Spiel mit vollem Enthusiasmus und ohne Rückhalt zu spielen setzt eine soziale Position voraus, mit der nicht nur eine Disposition zu intellektueller Hingabe einhergeht, sondern auch die Möglichkeit, damit einhergehende Einbußen oder Verluste habituell zu verklären und/oder sozioökonomisch abzufedern und zu kompensieren (vgl. die Ausführungen zur scholastischen Sicht, z.B. in Bourdieu 1998b, 2001).

Bisher hier zeigt sich also: Wissenschaftliche und institutionelle Meriten sowie der Fleiß und die Hingabe, durch die sie begründet sind, gehören zu den charakteristischen Maßstäben wissenschaftlicher Subjekte. Weil es sich dabei um Zuschreibungen handelt, die über alle im Sample enthaltenen Disziplinen und Länder hinweg sowie zu allen Zeitpunkten getätigt werden, ist anzunehmen, dass dies Ansprüche sind, denen jedes Subjekt - mindestens in einem Nachruf - genügen muss. Neben diesen allgemeingültigen Punkten können auch Kriterien und Maßstäbe unterschieden werden, die zwischen Ländern, Disziplinen und Phasen variieren.

\subsection{Talent, Internationalität und Quantifizierung}

Neben Universalismen, die im Sample keine spezifische Verteilung nach Ländern, Phasen oder Disziplinen aufweisen, lassen sich in Nachrufen auch Zuschreibungen rekonstruieren, die genau solche Variationen aufweisen. Eine im gesamten Sample zu findende, aber vor allem in deutschen Nachrufen verbreitete Alternative zu Narrativen des Fleißes und der Hingabe ist die Zuschreibung von Talent. Wissenschaftliche Subjekte werden dann an ihrer natürlichen Begabung gemessen, die akademische Profession angemessen auszuüben. Wenn Lebensläufe in deutschen Nachrufen unter Berufung auf Talent plausibilisiert werden, dann verweisen Nachrufe auf eine „,natürliche Fähigkeit“ (Aubin 1967, S. 575) zum akademischen Lehrer, auf eine „Berufung als Forscherin“ (Fouquet 2000, S. 2), auf ein „untrügliches Gefühl“ (Kellenbenz 
1965, S. 126) für Untersuchungsgegenstände oder auf ein „genuines Verständnis“ (Gollwitzer 1967, S. 297) für die akademische Welt.

Nicht selten kündigt sich eine solche Begabung bereits in jungen Jahren an, so dass die Idee der Vorherbestimmung für eine akademische Karriere eine typische Variation ist. Karrieren haben sich in dieser Perspektive unvermeidlich, fast schicksalshaft eingestellt. Biographische Vorherbestimmung wird zum Beispiel angedeutet, wenn ein Physiker vom „Geheimnis dieser [Röntgen-]Strahlen [...] nicht mehr losgelassen“ wurde, seitdem er ,als Schüler von den Arbeiten Röntgens erfahren und selbst versucht hatte, mit den unbekannten Strahlen zu experimentieren“ (Brüche 1960, S. 257), wenn sich bereits in der Dissertation Fragestellungen ankündigen, die einen Forscher dann „sein Leben lang beschäftigten“ (Luckmann 1961, S. 769) oder wenn eine Historikerin seit dem ,5. Semester fasziniert“ war von der „Geschichte des europäischen Städtewesens.“ (Fouquet 2000, S. 1) Rationalisiert wird Talent mit charakterlichen Zuschreibungen von Neugier (Schwarz 1999, S. 595; Schwengel 2002, S. 614), Kreativität (Berghoff 1999, S. 308; Steinmetz 2006, S. 427) oder einer akademischen Unangepasstheit (Hurrelmann 1987, S. 397; Bös 2007, S. 369), die ihre Kenntnis der Regeln des akademischen Feldes gerade dadurch unter Beweis stellt, dass sie spielerisch mit ihnen umgeht. Narrative des natürlichen Talents finden sich durchaus auch in britischen oder US-amerikanischen Nachrufen. Hervorgehobene Bedeutung hat dieser Maßstab aber in erster Linie für deutsche Wissenschaftssubjekte.

Das in deutschen Nachrufen relativ häufiger vorkommende Narrativ der natürlichen Begabung stellt eine weitere Verschleierung von Selektionsmechanismen dar. So wie meritokratische Maßstäbe des Fleißes und der Hingabe Kontingenzen und sozialstrukturelle Determinanten ausblenden, scheint die Idee einer natürlichen Begabung auf humanistischen Ideen wie der ganzheitlichen Bildung des Menschen an der Wissenschaft und der wechselseitigen Durchdringung von Individuum und Forschungstätigkeit zu basieren (Hamann 2014; Ricken 2006). Erfolg ist auf diese Weise immer schon in der Persönlichkeit des Subjekts angelegt. Er kann nicht erarbeitet, sondern nur erweckt werden. Dass die Orientierung an „Talent“ in Deutschland relativ häufiger als in den USA oder UK zu beobachten ist, mag nicht nur am neuhumanistischen Fundament liegen, auf dem die moderne Wissenschaft in Deutschland fußt, sondern auch an der besonders zentralen Stellung der meritokratischen Ideologie des American Dream (McNamee und Miller 2004), einer vor allem im angloamerikanischen Raum verbreiteten Erzählung, nach der Erfolg prinzipiell jeder und jedem offen steht, solange sie oder er nur hart genug arbeitet. 
Doch Bewertungsmaßstäbe für wissenschaftliche Subjekte variieren nicht nur zwischen Ländern, sondern auch im Zeitverlauf. Die auffälligste Entwicklung von den 1960ern bis in die 2000er Jahre ist die immer häufiger werdende Zuschreibung von „Internationalität“. Das Attribut wird dabei in unterschiedlichen Formen konstruiert. Es kann erstens die Form internationaler Anerkennung und Verdienste erhalten, die den Verstorbenen zum Beispiel als „respected on the international stage“ (Roberts 2007, S. 613) markieren oder feststellen, dass seine „Wirksamkeit längst transnational war, ehe die entsprechenden Begriffe in Mode kamen“ (Rehberg 2003, S. 821). Eine zweite Variante des Maßstabs Internationalität ist die Zuschreibung von Kosmopolitismus und internationaler Mobilität als Charaktermerkmalen, die Subjekte für ihren „,international outlook“ (Peacock 2005, S. 78) preisen oder dafür, dass „,background and orientation were quintessentially cosmopolitan“ (Foster 2013, S. 6). Drittens wird Internationalität über internationale Posten attestiert, etwa durch die Tätigkeit britischer Physiker an US-amerikanischen Observatorien (Kopal 1970, S. 147), durch Aufenthalte US-amerikanischer Soziologen als visiting professor in Oxford (Hauser 1959, S. 74) oder deutscher Historiker an der Harvard University (Schulz 2004, S. 419).

Internationalität ist ein Merkmal, dessen Bedeutung für die Bewertung akademischer Subjekte nicht nur im Zeitverlauf stark zunimmt. Es variiert auch nach Disziplinen: Während Nachrufe auf Physiker häufig auf deren Internationalität hinweisen, wird dieses Attribut in der Geschichtswissenschaft eher selten mobilisiert (siehe zu diesen Befunden auch Hamann und Zimmer 2016).

Die Ergebnisse zeigen, dass Internationalität von zunehmender Bedeutung für die Anerkennung akademischer Subjekte ist. Dieser Befund stellt nicht nur einen Beitrag zur umfangreichen Forschung über die Internationalisierung des Hochschul- und Wissenschaftssektors (Kosmützky 2015) und insbesondere zu Studien über die Internationalisierung von akademischen Kulturen und Praktiken dar (Altbach 2013; Huang et al. 2014). Deutlich wird auch, dass „Internationalität“ erstens nicht nur ein objektives Merkmal wissenschaftlicher Karrieren ist, sondern auch als ein konstruierter Wert verstanden werden muss, und dass der Aufstieg dieses Werts zweitens nicht in allen Ländern und Disziplinen gleich ausgeprägt ist.

Akademische Subjekte werden nicht nur an ihren akademischen und institutionellen Verdiensten, an ihrem Fleiß, ihrem Talent oder ihrer Internationalität gemessen. Entscheidend ist die dazu gewissermaßen quer liegende Frage, wie akademische Errungenschaften konzipiert und beschrieben werden. So können Verdienste und Leistungen über Forschungsinhalte begründet werden. Hervorgehoben werden dann beispielsweise bestimmte Beiträge von Publikationen. 
Ein Buch mag „[e]in wichtiges Buch für die empirische Migrationsforschung“ (Bös 2007, S. 368) sein oder zentrale Themen auf eine neue Weise besetzt haben: „In his last published book [,the] first chapter offered a brief, lucid, and thought-provoking answer to the question, What is the History of Ideas?“ (Wiener 1980, S. 153f). Diese auf Forschungsinhalte referierende Form der Darstellung von Leistungen kann bis zur ausführlichen Paraphrasierung von Büchern oder Artikeln gehen. Im Sample ist dies vor allem in älteren Nachrufen und vor allem in geschichtswissenschaftlichen Nachrufen der Fall.

Immer häufiger wird jedoch eine andere Form der Konzeption und Beschreibung von Leistungen. Im Fokus steht dann nicht mehr nur (inhaltliche) Qualität, sondern auch Quantität. Der Grund dafür, dass ein Verstorbener von seinen Peers anerkannt war und ist, können dann beispielsweise Zitationszahlen sein: „his works on science, for example, are still highly cited by any measure.” (Alexander 2006, o.S.) Akademische Subjekte werden geehrt auf der Grundlage, dass sie ,author of 355 publications“ (Wolfe 2010, S. 1255) waren oder „,more than 100 scientific papers“ (Walker et al. 1984, S. 535) publiziert haben. Typischerweise wird eine Publikation dann nicht mehr (nur) durch inhaltliche Qualitäten hervorgehoben, sondern weil sie ,has turned out to be [his] most frequently cited paper.“ (Hoffman 2009, S. 102) Hier zeigt sich eine deutliche Verschiebung von qualitativen in Richtung quantitativer Leistungsmerkmale. Diese Veränderung ist nicht nur im Zeitverlauf erkennbar. Im Ländervergleich spielt die Quantifizierung von Leistungen in deutschen Nachrufen eine deutlich geringere Rolle als in US-amerikanischen Nachrufen. Im Disziplinenvergleich nehmen quantifizierte Leistungen in Nachrufen auf Physiker deutlich mehr Raum ein als ein Nachrufen auf Historiker.

Die steigende Bedeutung zählbarer Leistungsmerkmale in akademischen Nachrufen spiegelt die zentrale Stellung verschiedenster Maßzahlen in der Governance von Universitäten und ihrem Personal wider: Zitationen, quantitative Modelle des Workloads, numerische Erfassungen von Lehrqualität, Drittmittel als Leistungsindikatoren oder Universitätsrankings sind Teil einer umfassenden Audit-Kultur (Power 1997), deren Datensammlungen zusehends nicht nur Märkte imitieren, sondern sie als neue Organisationsform tatsächlich hervorbringen (Burrows 2012; Lorenz 2015). Die in Nachrufen rekonstruierbare Quantifizierung akademischer Leistungen erscheint vor diesem Hintergrund eingebettet in eine neoliberale Herrschaft der Zahlen (Angermuller und Maeße 2015; Porter 1995; siehe auch den Beitrag von Münch in diesem Band), die ihren Weg in akademische Subjektivierungspraktiken gefunden hat.

Es kann somit festgehalten werden: Während wissenschaftliche und institutionelle Meriten sowie Fleiß und Hingabe universelle Maßstäbe zu sein scheinen, entlang derer wissenschaftliche 
Subjekte in allen im Sample enthaltenen Disziplinen, Ländern und Phasen hervorgebracht werden, sind natürliches Talent, Internationalität und quantifizierbare Leistungen Maßstäbe, die ein besonderes Gewicht in bestimmten Ländern haben oder deren Bedeutung im Zeitverlauf zunimmt. Zusammengenommen konnte eine Reihe von Zuschreibungen identifiziert werden, mit denen wissenschaftliche Subjekte konstituiert werden. Doch nicht alle Anforderungen werden so explizit formuliert. Der folgende Abschnitt beleuchtet Aspekte, an die Subjektivierungspraktiken gerade nicht anknüpfen, biographische Merkmale also, die von den diskursiven Zensurmechanismen des Feldes verschleiert und von der Illusio beschwiegen werden.

\subsection{Soziale Herkunft, Geschlecht, Familienstand und politische Positionen}

Bereits die vorangehenden Abschnitte haben deutlich gemacht, dass die Konstitution wissenschaftlicher Subjekte in Nachrufen sehr selektiv stattfindet. Im Sample zeigt sich, dass ganz bestimmte Merkmale und Zuschreibungen - beispielsweise Talent, Fleiß, wissenschaftliche Verdienste oder Internationalität - zu sehr spezifischen Subjektkonstruktionen verdichtet werden. Zu dieser Spezifik gehört auch, dass andere Merkmale dezidiert nicht erwähnt werden.

Das Geschlecht ist eine Determinante, deren Abwesenheit bei der Konstruktion von Subjektfiguren besonders auffällt (vgl. Bell und Yalom 1990; Paulitz 2012; siehe auch den Beitrag von Paulitz und Prietl in diesem Band). Die Mehrzahl der 216 Nachrufe im Sample handelt von männlichen Wissenschaftlern (93 Prozent) und ist von männlichen Autoren verfasst (95 Prozent). Dabei wird jedoch das männliche Geschlecht überhaupt nicht explizit thematisiert, während strukturell unterrepräsentierte Frauen als solche nur Anerkennung finden, wenn ihr Geschlecht in eine meritokratische Erfolgsgeschichte eingebaut werden kann. Wissenschaftlerinnen überwinden ihr Geschlecht dann wie ein Hindernis; von einer Verstorbenen wird berichtet: "[she] overcame the disadvantages of her [...] gender to become an internationally recognized Professor" (Purvis 2008, S. 363). Insgesamt wird Geschlecht nicht als Einflussfaktor oder als erklärende Variable für den Verlauf akademischer Karrieren herangezogen. Der in Nachrufen beschriebene Erfolg wissenschaftlicher Subjekte steht in keinem sichtbaren Verhältnis zu ihrem (männlichen oder weiblichen) Geschlecht - ein Subjektivierungsmuster, das durch die ausgeprägte Unterrepräsentation von Wissenschaftlerinnen im Sample konterkariert wird.

Auch die soziale Herkunft von Wissenschaftlern hat sich als einflussreiche Determinante für den Verlauf akademischer Karrieren erwiesen (siehe auch die Beiträge von Möller und Graf in diesem Band). Doch auch dieser Aspekt findet nur äußerst selten in Nachrufen Erwähnung. In diesen seltenen Fällen wird die soziale Herkunft der Verstorbenen dann auch nicht umfassend 
in die biographische Erzählung integriert, sondern in einem nüchternen Stil dokumentiert, der sich vom rühmenden, schmeichelnden Duktus des Genres merklich abhebt. Nachrufe bekunden dann lediglich, dass der Vater eines Verstorbenen "a naval officer" (Hargens 1988, S. 572) oder “an economic historian” (Loftus 2001, S. 299) war, ohne mögliche Effekte auf den biographischen Verlauf zu reflektieren. In ganz wenigen Ausnahmefällen wird der sozialen Herkunft der Verstorbenen tatsächlich ein Einfluss zugestanden. Anders als bei der Determinante Geschlecht, wo sich dann aus dem vermeintlichen Nachteil eine meritokratische Gewinnergeschichte ergibt, findet bei der sozialen Herkunft eher eine vorteilhafte bildungsnahe Schicht Erwähnung. Ein Subjekt mag beispielsweise „,in einem vom Geiste Goethes geprägten Elternhaus aufgewachsen“ (Schramm 1968, S. 784), oder „vom bildungsbürgerlich-protestantischen Elternhaus geprägt" (Wehler 1985, S. 143) sein.

Nicht nur Geschlecht und soziale Herkunft werden in ihrer Bedeutung für akademische Karrieren und Biographien typischerweise verschleiert. Auch andere soziale Merkmale gehen kaum in die Subjektkonstruktion ein. So spielt der Familienstand von Verstorbenen nur selten eine Rolle. Zwar kann ein Wissenschaftler als "loving, devoted, dutiful husband and father" (Bebb 1983, S. 498) charakterisiert werden oder Wissenschaftler können, wie es besonders bei älteren Nachrufen noch häufiger der Fall ist, von der Unterstützung ihrer Ehefrauen profitieren (Kopal 1972, S. 265; Strasser 1986, S. 142). Doch diese vereinzelten Zuschreibungen übernehmen in den Texten keine tragende, die Subjektkonstruktion strukturierende Funktion. Ebenso können Wissenschaftlerinnen in politischer Hinsicht positioniert werden, wenn Nachrufe sie als nationalkonservativ (Gollwitzer 1967, S. 305), als Demokrat (Rehberg 2003, S. 819) oder Patriot (Peterson 1988, S. 248) charakterisieren oder ihr politisches Engagement hervorheben (zum Beispiel für atomare Abrüstung wie bei French 2008, S. 116). Auch solche politischen Zuschreibungen spielen im Sample eine marginale Rolle und sind nur in sehr wenigen Textstellen zu finden. Wie Geschlecht, soziale Herkunft oder familiale Merkmale sind auch politische Positionierungen kein systematischer Teil der Konstitution von Wissenschaftssubjekten.

Die Verschleierung dieser sozialen Determinanten mag zunächst überraschen; insbesondere in einem vermeintlich dokumentarisch-biographischen Genre wie Nachrufen, und insbesondere weil der Forschungsstand zu wissenschaftlichen Karrieren darauf verweist, dass weder Talent noch Fleiß allein ausreichen, um akademische Trajektorien zu erklären (Long und Fox 1995). Neben sogenannten meritokratischen Faktoren haben sich der Familienstand (Hess et al. 2011; Wolf-Wenderl et al. 2004), politische Einstellungen (Gross und Fosse 2012; Klein et al. 2005), 
nicht zuletzt Geschlecht (Rossiter 1993; Uhly et al. 2015) und soziale Herkunft (Graf 2015; Kennelly et al. 1999; Laurison und Friedman 2015; Möller 2015), aber auch die Einbindung in Netzwerke (Lutter und Schröder 2014) als einflussreiche Determinanten für wissenschaftliche Karrieren erwiesen. Trotz dieser Befunde wird solchen nicht-meritokratischen Einflussfaktoren nur sehr wenig Raum bei der Hervorbringung von Wissenschaftssubjekten in Nachrufen eingeräumt. Hier zeigt sich empirisch, wie Nachrufe als Arenen symbolischer Herrschaft fungieren: Subjektpositionen werden im Rahmen ihrer Konsekration in einem sozialen Vakuum platziert, ihre Position im sozialen Raum wird verkannt. Gerade nicht-akademische Formen der gesellschaftlichen Einbettung des wissenschaftlichen Personals, die nicht nur sozialstrukturell und habituell offensichtlich, sondern auch gut erforscht sind, werden auf diese Weise verschleiert. Weniger überraschend ist diese selektive Konstruktion angesichts dessen, was Bourdieu (1998b, S. 205f) epistemische Doxa nennt: die Tendenz von Wissenschaftlerinnen und Wissenschaftlern, die ökonomischen und sozialen Voraussetzungen ihrer scholastischen Haltung nicht mitzudenken.

\section{Diskussion und Fazit: Effekte der Subjektivierung in akademischen Nachrufen}

Welche Schlüsse können aus den Befunden gezogen werden? Zunächst ist deutlich geworden, dass wissenschaftliche Subjekte in Nachrufen auf eine spezifische Form konstituiert werden. Sie werden ganz überwiegend in einem sehr positiven Licht dargestellt. Im Vergleich mit in Zeitungen veröffentlichten Nachrufen auf Personen des öffentlichen Lebens, die durchaus Raum für Kritik oder biographische Brüche lassen (Fowler 2007, S. 63), sind in Fachzeitschriften veröffentlichte, von Peers verfasste Nachrufe auf Wissenschaftlerinnen durch ihre geradezu schmeichelhafte Darstellung charakterisiert. Meine Analyse hat gezeigt, dass zu diesem Zweck wissenschaftliche und institutionelle Verdienste, der Fleiß und die Hingabe der Verstorbenen sowie ihr Talent oder ihre internationale Ausrichtung hervorgehoben werden. Bei akademischen Nachrufen handelt es sich demnach um eine Praxis der Subjektivierung, bei der ein verdientes Mitglied der jeweiligen Gemeinschaft konsekriert wird. Konsekrationen verleihen eine sozial verbindliche Weihe und lassen die Träger zu Geweihten werden. Während Bourdieu (1992) unter Konsekrationsinstanzen beispielsweise Verlage, Buchreihen oder Forschungsinstitute fasst, beschreibt Münch (2008) in ähnlicher Weise die systematische Evaluation eines Fachbereichs als eine Instanz der Statuszuweisung. Der vorliegende Beitrag versteht Nachrufe als Instanzen, die bestimmte Subjekte und ihre Biographien konstruieren und kanonisieren und damit sichtbar machen, welche Erwartungen an akademische Lebensläufe gestellt werden. 
Die Figuren, auf die Nachrufe rekurrieren, geben den Blick frei auf allgemeinere Regeln des Feldes und seine ansonsten meist beschwiegenen Subjekte. Darstellungen erfolgreicher Biographien in der Wissenschaft schreiben bestimmte Verdienste und Eigenschaften zu und blenden soziale Faktoren aus (vgl. Beaufaÿs 2003). Ihre volle Wirkung als Konsekrationsinstanzen entfalten Nachrufe, weil sie nicht nur verstorbene Subjekte konstituieren, sondern vor allem für noch lebende Wissenschaftler wirken. Mit ihrem Aufforderungscharakter stellen sie eine potenziell einschüchternde, in jedem Fall disziplinierende Zugangshürde für das wissenschaftliche Personal dar. Diesem mag die glanzvolle Konstruktion von idealisierten Subjektfiguren als Druck erscheinen, ihre eigene Lebenspraxis an jenen Maßstäben auszurichten, die durch den Konsekrationsakt ausbuchstabiert werden. Wissenschaftler, die über ihre sozialen Voraussetzungen und ihre Lebensweise nicht symbolisieren können, dass sie wissenschaftliche und institutionelle Verdienste vereinen, dass sie hart und hingebungsvoll arbeiten und dass sie international aufgestellt sind, werden symbolisch diskriminiert und marginalisiert. Für bereits etablierte Wissenschaftlerinnen hingegen generieren Nachrufe symbolisches Kapital, weil sie im Konsekrationsakt als Priester ihrer Gemeinschaft auftreten und Merkmale einer legitimen akademischen Lebensführung nach den geltenden Regeln des Feldes definieren können (vgl. Beaufaÿs 2015). Konstituiert und geweiht werden daher nicht nur verstorbene, sondern auch noch lebende Subjekte. Ihre Karrieren und Werke haben nach den gleichen Regeln Bedeutung erlangt, die im Nachruf am Beispiel der Biographie eines verstorbenen Mitglieds der Gemeinschaft durchdekliniert werden. Die Wahrheit, der sich alle Beteiligten verschreiben müssen, (v-)erklärt Erfolg - und demzufolge auch Misserfolg - mit subjektivem Talent und Fleiß sowie mit wissenschaftlichen und institutionellen Meriten, nicht aber mit Merkmalen wie sozialer Herkunft oder Geschlecht.

Als überzeugend, ja geradezu folgerichtig und zwingend erscheint die Konsekration akademischer Subjekte dann aufgrund biographischer Narrative (vgl. Hamann 2016). Sie verleihen wissenschaftlichen Lebensläufen den Charakter einer gerichteten, chronologischen Sequenz (Bourdieu 1998a; Wacquant 2000). Es ist diese von einer empathischen Subjektidee suggerierte Einheit und Ganzheit biographischer Artefakte, durch die Konsekrationsakte als solche verkannt und verschleiert werden. Von zugeschriebenen Leistungen und Qualitäten wird dann nicht mehr auf objektive Feldstrukturen geschlossen; sie werden stattdessen durch die Idee des „Subjekts“ zu einer Einheit zusammengezogen und erhalten den Charakter des subjektiv Erlebten. In diesem Punkt haben Narrative des Fleißes und der Hingabe wie auch Erzählungen des Talents und der Begabung den gleichen Effekt: Aus dem fast sprichwörtlichen akademischen Hazard (Weber 1922), aus prekären Laufbahnen, Krisen und Sackgassen (Enders 2003; Kreckel 
und Zimmermann 2014) werden lineare und im Rückblick planvolle und plausible Helden- und Erfolgsgeschichten, an denen Wissenschaftler gemessen werden.

Worum geht es also in einem wissenschaftlichen Spiel, dessen Biographien und Karrieren auf diese Weise geregelt sind und das sich seine Subjekte so konstituiert? Rufen wir uns in Erinnerung, auf welche spezifischen Aspekte die hochselektive Darstellung von Wissenschaftssubjekten in Nachrufen Bezug nimmt. Es geht vor allem um harte Arbeit, um wissenschaftliche und institutionelle Verdienste, aber auch um Talent und Internationalität. Andere biographische Aspekte wie soziale Herkunft, Geschlecht, politische Positionen, private und familiale Merkmale treten systematisch in den Hintergrund. Nachrufe machen als Konsekrationsinstanzen damit genau jenes „Bündel von ökonomischen und sozialen Bedingungen“ (Bourdieu 1998b, S. 205) unsichtbar, das erst die Voraussetzung akademischer Tätigkeit ist. Mit den Kriterien die sie an Subjekte anlegen und entlang derer sie Biographien legitimieren, reproduzieren Nachrufe ein allgemeines, professionell-professorales Ethos. Sie perpetuieren die Illusio einer individuellen Agency, die Lebensläufe subjektiviert, Erfolg - und Misserfolg - internalisiert und legitime Karrieren autonomisiert, während externe Determinanten und sozioökonomische Einflussfaktoren ausgeblendet werden. Die Herrschaftsstrukturen des Feldes werden durch diese von Nachrufen vorgenommene Invisibilisierung imprägniert. Die Ausgangsfrage meines Beitrags war, wie wissenschaftliche Sprecher entstehen und wie Positionen besetzt werden, von denen aus auch in anderen Gesellschaftsbereichen wissenschaftliche „Wahrheit“" gesprochen werden kann. Die Autorität und Glaubwürdigkeit, mit der Wissenschaftssubjekte ihre Expertisen, Claims, Beratungen und Interventionen in gesellschaftlichen Kontexten platzieren, basiert auch auf einem Vertrauen in wissenschaftsinterne Mechanismen der kritisch-rationalen Leistungsund Qualitätskontrolle. Die Praktiken der feldinternen Konstitution von Sprecherinnen haben gezeigt, dass es notwendig ist, diese Quellen der wissenschaftlichen Autorität zu hinterfragen.

\section{Literatur}

Abbott, A. (1995). Things of Boundaries. Social Research, 62(4), 857-882.

Alkemeyer, T. (2013). Subjektivierung in sozialen Praktiken. Umrisse einer praxeologischen Analytik. In T. Alkemeyer, G. Budde, \& D. Freist (Hrsg.), Selbst-Bildungen. Soziale und kulturelle Praktiken der Subjektivierung (S. 33-68). Bielefeld: transcript.

Altbach, P. G. (2013). The International Imperative in Higher Education Rotterdam: SensePublishers.

Althusser, L. (1977). Ideologie und ideologische Staatsapparate. Aufsätze zur marxistischen Theorie. Hamburg: VSA.

Angermuller, J. (2013). How to become an academic philosopher. Academic discourse as multileveled positioning practice. Sociología histórica, 2013(2), 263-289.

Angermuller, J. (2014). Poststructuralist Discourse Analysis. Subjectivity in Enunciative Pragmatics. Houndsmill, Basingstoke: Palgrave Macmillan. 
Angermuller, J., \& Maeße, J. (2015). Regieren durch Leistung. Zur Verschulung des Sozialen in der Numerokratie. In A. Schäfer, \& C. Thompson (Hrsg.), Leistung. Reihe: Pädagogik Perspektiven (S. 61-108). Paderborn: Schöningh.

Baert, P. (2012). Positioning Theory and Intellectual Interventions. Journal for the Theory of Social Behaviour, 42(3), 304-324.

Bamberg, M. G. W. (2010). Who am I? Narration and its contribution to self and identity. Theory \& Psychology, 21(1), 1-22.

Beaufaÿs, S. (2003). Wie werden Wissenschaftler gemacht? Beobachtungen zur wechselseitigen Konstitution von Geschlecht und Wissenschaft. Bielefeld: transcript.

Beaufaÿs, S. (2015). Die Freiheit arbeiten zu dürfen. Akademische Laufbahn und legitime Lebenspraxis Beiträge zur Hochschulforschung, 37(3), 40-59.

Becher, T., \& Trowler, P. (2001). Academic Tribes and Territories: Intellectual Enquiry and the Cultures of Disciplines. Philadelphia: Open University Press.

Bell, S. G., \& Yalom, M. (Eds.). (1990). Revealing Lives: Autobiography, Biography, and Gender. Albany, NY: State University of New York Press.

Ben-David, J. (1977). Centers of Learning: Britain, France, Germany, United States. New York: McGraw-Hill Book Company.

Bernhard, S. (2014). Identitätskonstruktionen in narrativen Interviews. Ein Operationalisierungsvorschlag im Anschluss an die relationale Netzwerktheorie. Forum Qualitative Sozialforschung, 15(3), Art 1.

Bonsu, S. K. (2007). The Presentation of Dead Selves in Everyday Life: Obituaries and Impression Management. Symbolic Interaction, 30(2), 199-219.

Bonzi, S. (1992). Trends in research productivity among senior faculty. Information Processing \& Management, 1992(28), 111-120.

Bourdieu, P. (1992). Homo academicus. Frankfurt/M.: Suhrkamp.

Bourdieu, P. (1998a). Die biographische Illusion. In P. Bourdieu (Hrsg.), Praktische Vernunft. Zur Theorie des Handelns (S. 75-90). Frankfurt/M.: Suhrkamp.

Bourdieu, P. (1998b). Praktische Vernunft. Zur Theorie des Handelns. Frankfurt/M.: Suhrkamp.

Bourdieu, P. (2001). Meditationen. Zur Kritik der scholastischen Vernunft. Frankfurt/M.: Suhrkamp.

Bourdieu, P. (2004). Science of Science and Reflexivity. Cambridge: Polity Press.

Bröckling, U. (2007). Das unternehmerische Selbst. Soziologie einer Subjektivierungsform. Frankfurt/M.: Suhrkamp.

Burrows, R. (2012). Living with the h-index? Metric assemblages in the contemporary academy. The Sociological Review, 60(2), 355-372.

Butler, J. (1997). Excitable Speech. A Politics of the Performative. New York, London: Routledge.

Bytheway, B., \& Johnson, J. (1996). Valuing lives? Obituaries and the life course. Mortality, 1(2), 219234.

del Pierco, A., Zienkowski, J., \& Angermuller, J. (2014). Vom determinierten Akteur zum dezentrierten Subjekt. Strukturalismus, pragmatische und poststrukturalistische Perspektiven der Diskurstheorie im Dialog. In J. Angermuller, M. Nonhoff, E. Herschinger, F. Macgilchrist, M. Reisigl, J. Wedl, et al. (Hrsg.), Diskursforschung. Ein interdisziplinäres Handbuch, Bd. 1: Theorien, Methodologien und Kontroversen (S. 364-385). Bielefeld: transcript.

Dietz, J. S., Chompalov, I., Bozeman, B., Lane, E. O. N., \& Park, J. (2000). Using the curriculum vita to study the career paths of scientists and engineers: An exploratory assessment. Scientometrics, 49(3), 419-422.

Echterhölter, A. (2012). Schattengefechte. Genealogische Praktiken in Nachrufen auf Naturwissenschaftler (1710-1860). Göttingen: Wallstein Verlag.

Eid, M. (2002). The World of Obituaries: Gender Across Cultures and Over Time. Detroit, MI: Wayne State University Press.

Emmel, N. (2013). Sampling and Choosing Cases in Qualitative Research. Los Angeles: SAGE.

Enders, J. (2003). Flickwerkkarrieren und Strickleitern in einer prekären Profession. Ein Beitrag zur Nachwuchspolitik an den Hochschulen. In R. Hitzler, \& M. Pfadenhauer (Hrsg.), Karrierepolitik. Beiträge zur Rekonstruktion erfolgsorientierten Handelns (S. 253-262). Opladen: Leske + Budrich. 
These are proofs of the final publication available at Springer:

https://link.springer.com/chapter/10.1007/978-3-658-14900-0_4

Engler, S. (2001). »In Einsamkeit und Freiheit«? Zur Konstruktion der wissenschaftlichen Persönlichkeit auf dem Weg zur Professur. Konstanz: UVK.

Etzemüller, T. (2013). Der 'Vf.' als Subjektform. Wie wird man zum 'Wissenschaftler' und (wie) lässt sich das beobachten? In T. Alkemeyer, G. Budde, \& D. Freist (Hrsg.), Selbst-Bildungen. Soziale und kulturelle Praktiken der Subjektivierung (S. 175-196). Bielefeld: transcript.

Etzemüller, T. (2015). Ins »Wahre« rücken. Selbstdarstellung im Wissenschaftsbetrieb. Merkur, 69(797), 31-47.

Foucault, M. (1976). Überwachen und Strafen. Die Geburt des Gefängnisses. Frankfurt/M.: Suhrkamp.

Foucault, M. (2010). The Government of Self and Others. Lectures at the Collège de France 1982-1983. New York: Palgrave Macmillan.

Fowler, B. (2005a). Collective Memory and Forgetting: Components for a Study of Obituaries. Theory, Culture \& Society, 22(6), 53-72.

Fowler, B. (2005b). Mapping the obituary: Notes towards a Bourdieusian interpretation. The Sociological Review, 52(2), 148-171.

Fowler, B. (2007). The Obituary as a Collective Memory. New York: Routledge.

Fowler, B., \& Bielsa, E. (2007). The lives we choose to remember: a quantitative analysis of newspaper obituaries. The Sociological Review, 55(2), 203-226.

Gengnagel, V., \& Hamann, J. (2014). The Making and Persisting of Modern German Humanities. Balancing Acts between Autonomy and Social Relevance. In R. Bod, J. Maat, \& T. Weststeijn (Hrsg.), The Making of the Humanities III. The Modern Humanities (S. 641-654). Amsterdam: Amsterdam University Press.

Giroux, H. A. (2014). Neoliberalism's War on Higher Education. Chicago: Haymarket Books.

Gispen, K. (1989). New Profession, Old Order: Engineers and German Society, 1815-1914. Cambridge: University of Cambridge Press.

Graf, A. (2015). Die Wissenschaftselite Deutschlands. Sozialprofil und Werdegänge zwischen 1945 und 2013. Frankfurt/M.: Campus.

Gross, N. (2002). Becoming a Pragmatist Philosopher: Status, Self-Concept, and Intellectual Choice. American Sociological Review, 67(1), 52-76.

Gross, N., \& Fosse, E. (2012). Why are professors liberal? Theory and Society, 41(2), 127-168.

Guetzkow, J., Lamont, M., \& Mallard, G. (2004). What is Originality in the Humanities and the Social Sciences? American Sociological Review, 69(2), 190-212.

Gusfield, J. (1976). The Literary Rhetoric of Science: Comedy and Pathos in Drinking Driver Research. American Sociological Review, 1976(41), 16-34.

Gustin, B. H. (1973). Charisma, Recognition, and the Motivation of Scientists. American Journal of Sociology, 78(5), 1119-1134.

Häder, M. (2010). Empirische Sozialforschung. Eine Einführung. Wiesbaden: VS Verlag.

Hadjar, A. (2008). Meritokratie als Legimitationsprinzip. Die Entwicklung der Akzeptanz sozialer Ungleichheit im Zuge der Bildungsexpansion. Wiesbaden: VS.

Hahn, A. (1988). Biographie und Lebenslauf. In H.-G. Brose, \& B. Hildenbrand (Hrsg.), Vom Ende des Individuums zur Individualität ohne Ende (S. 91-105). Opladen: Leske + Budrich.

Halsey, A. H. (2004). A History of Sociology in Britain: Science, Literature, and Society. Oxford: Oxford University Press.

Hamann, J. (2014). Die Bildung der Geisteswissenschaften. Zur Genese einer sozialen Konstruktion zwischen Diskurs und Feld. Konstanz: UVK.

Hamann, J. (2015). Posthumous (E)valuation. Research Biographies in US Sociology, as Reflected in Academic Obituaries. Timelines - Newsletter of the ASA Section History of Sociology, 2015(24), $10-13$.

Hamann, J. (2016). 'Let us salute one of our kind'. How academic obituaries consecrate research biographies. Poetics, DOI :10.1016/j.poetic.2016.02.005, 1-14.

Hamann, J., \& Zimmer, L. M. (2016). The Internationality Imperative in Academia. The Ascend of Internationality as an Academic Virtue. Manuskript im Review.

Hess, J., Rusconi, A., \& Solga, H. (2011). »Wir haben dieselben Ziele ...« - Zur Bedeutung von Paarkonstellationen und Disziplinenzugehörigkeit für Karrieren von Frauen in der Wissenschaft. In W. Cornelißen, A. Rusconi, \& R. Becker (Hrsg.), Berufliche Karrieren von Frauen: Hürdenläufe in Partnerschaft und Arbeitswelt (S. 65-104). Wiesbaden: VS. 
These are proofs of the final publication available at Springer:

https://link.springer.com/chapter/10.1007/978-3-658-14900-0_4

Hirschauer, S. (2010). Editorial Judgements: A Praxeology of 'Voting' in Peer Review. Social Studies of Science, 40(1), 71-103.

Huang, F., Finkelstein, M., \& Rostan, M. (Eds.). (2014). The Internationalization of the Academy. Changes, Realities and Prospects. Dordrecht: Springer.

Kagan, J. (2009). The Three Cultures: Natural Sciences, Social Sciences, and the Humanities in the 21st Century. Cambridge: Cambridge University Press.

Kelle, U., \& Kluge, S. (2010). Vom Einzelfall zum Typus. Fallvergleich und Fallkontrastierung in der qualitativen Sozialforschung. Wiesbaden: VS.

Kennelly, I., Misra, J., \& Karides, M. (1999). The Historical Context of Gender, Race, \& Class in the Academic Labor Market. Race, Gender \& Class, 6(3), 125-155.

Klein, D. B., Stern, C., \& Western, A. (2005). Political diversity in six disciplines. Academic Questions, 18(1), 40-52.

Kohli, M. (1981). 'Von uns selber schweigen wir.' Wissenschaftsgeschichte aus Lebensgeschichten. In W. Lepenies (Hrsg.), Geschichte der Soziologie. Studien zur kognitiven, sozialen und historischen Identität einer Disziplin, 4 Bde. (S. 428-465). Frankfurt/M.: Suhrkamp.

Koschorke, A. (2012). Wahrheit und Erfindung: Grundzüge einer Allgemeinen Erzähltheorie. Frankfurt/M.: Fischer.

Kosmützky, A. (2015). Transcending Borders and Traversing Boundaries - A Systematic Review of the Literature on Transnational, Offshore, Cross-border and Borderless Higher Education. Journal of Studies in International Education, 2015(9), Online first: DOI: 10.1177/1028315315604719.

Kreckel, R., \& Zimmermann, K. (2014). Hazard oder Laufbahn. Akademische Karrierestrukturen im internationalen Vergleich. Leipzig: Akademische Verlagsanstalt.

Kresta, R. (1996). »Nachrufe« in englischen und deutschen Fachzeitschriften in der Soziologie. Fachsprache, 1996(18), 118-137.

Lamont, M. (1987). How to Become a Dominant French Philosopher: The Case of Jacques Derrida. The American Journal of Sociology, 93(3), 584-622.

Lamont, M. (2009). How Professors Think. Inside the Curious World of Academic Judgement. Cambridge, MA, London: Harvard University Press.

Lamont, M., Kaufman, J., \& Moody, M. (2000). The Best of the Brightest: Definitions of the Ideal Self Among Prize-Winning Students. Sociological Forum, 15(2), 187-224.

Lamont, M., \& Molnár, V. (2002). The Study of Boundaries in the Social Sciences. Annual Review of Sociology, 28(2002), 167-195.

Laurison, D., \& Friedman, S. (2015). Introducing the Class Ceiling: Social Mobility into Britain's Elite Occupations. LSE Sociology Department Working Paper Series. London: LSE Academic Publishing.

Long, G. L. (1987). Organizations and Identity: Obituaries 1856-1972. Social Forces, 65(4), 964-1001.

Long, S. J., \& Fox, M. F. (1995). Scientific Careers: Universalism and Particularism. Annual Review of Sociology, 21(1995), 45-71.

Lorenz, C. (2015). The Metrification of 'Quality' and the Fall of the Academic Profession. Oxford Magazine, 355(Noughth Week), 7-11.

Lutter, M., \& Schröder, M. (2014). Who Becomes a Tenured Professor, and Why? Panel Data Evidence from German Sociology, 1980-2013. Research Policy, 45(5), 999-1013.

Macfarlane, B., \& Chan, R. Y. (2014). The last judgement: exploring intellectual leadership in higher education through academic obituaries. Studies in Higher Education, 39(2), 294-306.

Maeße, J. (2015a). Economic Experts. A Discursive Political Economy of Economics. Journal of Multicultural Discourses, 10(3), 279-305.

Maeße, J. (2015b). Eliteökonomen. Wissenschaft im Wandel der Gesellschaft. Wiesbaden: VS Verlag für Sozialwissenschaften.

Maeße, J., \& Hamann, J. (2016). Die Universität als Dispositiv. Die gesellschaftliche Einbettung von Bildung und Wissenschaft aus diskurstheoretischer Perspektive. Zeitschrift für Diskursforschung, 2016(1), 29-50.

McNamee, S. J., \& Miller, R. K. J. (2004). The Meritocracy Myth. Langham, Md. et al.: Rowman \& Littlefield.

Merton, R. K. (1973). The normative structure of science. In R. K. Merton (Hrsg.), The Sociology of Science (S. 267-278). Chicago: University of Chicago Press. 
These are proofs of the final publication available at Springer:

https://link.springer.com/chapter/10.1007/978-3-658-14900-0 4

Mialet, H. (1999). Do Angels Have Bodies? Two Stories About Subjectivity in Science: The Cases of William X and Mister H. Social Studies of Science, 29(4), 551-581.

Möller, C. (2015). Herkunft zählt (fast) immer. Soziale Ungleichheiten unter Universitätsprofessorinnen und -professoren. Weinheim, Basel: Beltz Juventa.

Moore, K., Kleinman, D. L., Hess, D., \& Frickel, S. (2011). Science and neoliberal globalization: a political sociological approach. Theory and Society, 40(5), 505-532.

Münch, R. (2008). Stratifikation durch Evaluation. Mechanismen der Konstruktion und Reproduktion von Statushierarchien in der Forschung. Zeitschrift für Soziologie, 37(1), 60-80.

Musselin, C. (2009). The market for academics. New York: Routledge.

Patton, M. Q. (2002). Qualitative Research \& Evaluation Methods. Thousand Oaks: SAGE.

Paulitz, T. (2012). 'Hegemoniale Männlichkeiten' als narrative Distinktionspraxis im Wissenschaftsspiel. Österreichische Zeitschrift für Soziologie, 2012(37), 45-64.

Paulitz, T., Goisauf, M., \& Zapusek, S. (2015). Work-Life-Balance + Wissenschaft = unvereinbar? Zur exkludierenden Vergeschlechtlichung einer entgrenzten Lebensform. Gender, 7(2).

Peter, T. (2014). Genealogie der Exzellenz. Weinheim: Beltz Juventa.

Porter, T. M. (1995). Trust in Numbers: The Pursuit of Objectivity in Science and Public Life. Princeton: Princeton University Press.

Power, M. (1997). The Audit Society. Rituals of Verification. Oxford: Oxford University Press.

Reckwitz, A. (2006). Das hybride Subjekt. Eine Theorie der Subjektkulturen von der bürgerlichen Moderne zur Postmoderne. Weilerswist: Velbrück.

Reckwitz, A. (2008). Subjekt. Bielefeld: transcript.

Ricken, N. (2006). Die Ordnung der Bildung. Beiträge zu einer Genealogie der Bildung. Wiesbaden: VS.

Rodler, C., Kirchler, E., \& Hölzl, E. (2001). Gender Stereotypes of Leaders: An Analysis of the Contents of Obituaries from 1974 to 1998. Sex Roles, 45(11-12), 827-843.

Rossiter, M. W. (1993). The Matthew Matilda Effect in Science. Social Studies of Science, 23(2), 325341.

Shortland, M., \& Yeo, R. (Eds.). (1996). Telling Lives in Science: Essays on Scientific Biography. Cambridge: Cambridge University Press.

Söderqvist, T. (2011). The Seven Sisters: Subgenres of Bioi of Contemporary Life Scientists. Journal of the History of Biology, 2011(44), 633-650.

Strauss, A. L., \& Corbin, J. M. (1990). Basics of Qualitative Research. Grounded Theory Procedures and Techniques. Newbury Park: Sage.

Tight, M. (2008). Dead academics: what can we learn about academic work and life from obituaries? London Review of Education, 6(2), 125-135.

Traue, B. (2010). Das Subjekt der Beratung. Zur Soziologie einer Psycho-Technik. Bielefeld: transcript.

Tsay, A., Lamont, M., Abbott, A., \& Guetzkow, J. (2003). From character to intellect: changing conceptions of merit in the social sciences and humanities, 1951-1971. Poetics, 2003(31), 2349.

Uhly, K., Visser, L. M., \& Zippel, K. (2015). Gendered patterns in international research collaborations in academia. Studies in Higher Education, 10.1080/03075079.2015.1072151, 1-23.

Wacquant, L. D. (2000). Academic Portraits: Autobiography and Scientific Censorship in American Sociology. In G. C. Kinloch, \& R. P. Mohan (Hrsg.), Ideology and the Social Sciences (S. 147157). Westport, London: Greenwood Press.

Weber, M. (1922). Wissenschaft als Beruf. In M. Weber (Hrsg.), Gesammelte Aufsätze zur Wissenschaftslehre (S. 524-555). Tübingen: J.C.B. Mohr.

Wolf-Wenderl, L. B., Twombly, S. B., \& Rice, S. (2004). The Two-Body Problem. Dual-Career-Couple Hiring Practices in Higher Education. Baltimore, MD: John Hopkins University Press.

Zimmermann, K. (2004). Berufungsspiele des wissenschaftlichen Feldes im Lichte des Konzepts »symbolische Gewalt«. In J. Ebrecht, \& F. Hillebrandt (Hrsg.), Bourdieus Theorie der Praxis. Erklärungskraft, Anwendung, Perspektiven (S. 139-151). Wiesbaden: VS. 
These are proofs of the final publication available at Springer:

https://link.springer.com/chapter/10.1007/978-3-658-14900-0 4

\section{Anhang: Im Text zitierte Nachrufe}

Alexander, J. C., Cole, J. R., \& Zelizer, V. (2006). Bernard Barber (1918-2006). ASA Footnotes, 34(8), o.S.

Aubin, H. (1967). Hektor Ammann. Vierteljahrschrift für Sozial- und Wirtschaftsgeschichte, 54(4), 572576.

Bebb, P. N. (1983). In Memoriam: Harold J. Grimm. The Sixteenth Century Journal, 14(4), 497-498.

Berghoff, H., \& Ziegler, D. (1999). Sidney Pollard (1925-1998). Vierteljahrschrift für Sozial- und Wirtschaftsgeschichte, 86(3), 305-308.

Bös, M. (2007). In memoriam Karl Otto Hondrich. Kölner Zeitschrift für Soziologie und Sozialpsychologie, 59(2), 367-369.

Brüche, E. (1960). Abschied von Max von Laue. Physikalische Blätter, 1960(5), 257-258.

Brüche, E. (1970). Josef Zähringer in memoriam. Physikalische Blätter, 26(8), 365-366.

Cobb, R. C. (1960). Georges Lefebvre. Past \& Present, 18(1), 52-67.

Conze, W. (1982). Erich Maschke (1900-1982). Vierteljahrschrift für Sozial- und Wirtschaftsgeschichte, 69(2), 300-301.

Foster, R. (2013). Eric Hobsbawm. Past \& Present, 218(1), 3-15.

Fouquet, G. (2000). Edith Ennen (1907-1999). Vierteljahrschrift für Sozial- und Wirtschaftsgeschichte, $87(1), 1-4$.

French, A. P. (2008). In Memoriam Philip Morrison. Physics in Perspective, 10(2008), 110-122.

Gollwitzer, H. (1967). Karl Alexander von Müller 1882-1964: Ein Nachruf. Historische Zeitschrift, 205(2), 295-322.

Hargens, L. L., \& Gieryn, T. F. (1988). Nicholas C. Mullins (1939-88). Social Studies of Science, 18(3), $572-573$.

Hauser, P. M. (1959). William Fielding Ogburn, 1886-1959. American Journal of Sociology, 65(1), 74.

Hauser, P. M. (1961). In Memoriam: Samuel Andrew Stouffer, 1900-1960. American Journal of Sociology, 66(4), 364-365.

Hoffman, G. L. (2009). Edwin E. Salpeter (1924-2008). Publications of the Astronomical Society of the Pacific, 121(876), 101-103.

Hurrelmann, K. (1987). Hans Haferkamp (15.9.1939-14.7.1987). Zeitschrift für Soziologie, 16(5), 395398.

Kellenbenz, H. (1965). Bruno Kuske: Das wirtschafts- und sozialgeschichtliche Werk Bruno Kuskes. Vierteljahrschrift für Sozial- und Wirtschaftsgeschichte, 52(1), 125-144.

Kopal, Z. (1972). In Memoriam Harlow Shapley. Astrophysics and Space Science, 1972(18), 259-266.

Koshiba, M., Melissinos, A. C., \& Savedoff, M. P. (2003). Morton Fischel Kaplon. Physics Today, 56(4), 81-82.

Lepsius, R. M. (1986). In memoriam Johannes Winckelmann (29. März 1900 - 21. November 1985). Kölner Zeitschrift für Soziologie und Sozialpsychologie, 1986(38), 414-416.

Loftus, D. (2001). Robert Q. Gray (1945-2001). History Workshop Journal, 2001(52), 299-303.

Luckmann, T. (1961). Nekrolog. Alfred Schütz. Kölner Zeitschrift für Soziologie und Sozialpsychologie, 1961(13), 768-770.

Münch, R. (1987). In memoriam Hans Haferkamp (15.09.1939-14.07.1987). Kölner Zeitschrift für Soziologie und Sozialpsychologie, 1987(39), 625-628.

Outhwaite, W. (1993). Tom Bottomore: Born 8.4.20. Died 9.12.92. Sociology, 27(3), 387-399.

Peacock, N. J., \& Christiansen, J. P. (2005). Rendel Sebastian Pease. Physics Today, 58(6), 78-79.

Purvis, J. (2008). Olive Banks (1923-2006): an appreciation. British Journal of Sociology of Education, 29(4), 363-368.

Rehberg, K.-S. (2003). In memoriam Erwin K. Scheuch. Kölner Zeitschrift für Soziologie und Sozialpsychologie, 2003(55), 819-821.

Roberts, I. (2007). In Memorium: Richard Kemp Brown. Work, Employment \& Society, 21(4), 613-614.

Schramm, P. E. (1968). Wolfram von den Steinen. Historische Zeitschrift, 207(3), 783-787.

Schulz, G. (2004). Wolfgang Zorn (1922-2004). Vierteljahrschrift für Sozial- und Wirtschaftsgeschichte, 91(4), 419-421.

Schwarz, H.-P. (1999). Nachruf auf Theodor Eschenburg. Vierteljahrshefte für Zeitgeschichte, 47(4), 593-600. 
These are proofs of the final publication available at Springer:

https://link.springer.com/chapter/10.1007/978-3-658-14900-0_4

Schwengel, H. (2002). In memoriam Heinrich Popitz. Kölner Zeitschrift für Soziologie und Sozialpsychologie, 2002(54), 614-615.

Steinmetz, W. (2006). Nachruf auf Reinhart Koselleck (1923-2006). Geschichte und Gesellschaft, 32(3), 412-432.

Strasser, H. (1986). Werner Stark - Gelehrter und Katholik: 1909-1985. Zeitschrift für Soziologie, 15(2), 141-145.

Stryker, S. (1968). Arnold M. Rose 1918-1968. The American Sociologist, 3(1), 60-61.

van Biesbroeck, G. (1961). Frank Elmore Ross. Quarterly Journal of the Royal Astronomical Society, 1961(2), 278.

Walker, A. B. C., Helliwell, R. A., \& Sturrock, P. A. (1984). John Marsh Wilcox. Quarterly Journal of the Royal Astronomical Society, 25(4), 534-536.

Wehler, H.-U. (1985). Nachruf auf Theodor Schieder: 11. April 1908-8. Oktober 1984. Geschichte und Gesellschaft, 11(1), 143-153.

Wiener, P. P. (1980). In Memoriam: George Boas (1891-1980). Journal of the History of Ideas, 41(3), 453-456.

Wolfe, A. M. (2010). Geoffrey R. Burbidge (1925-2010). Publications of the Astronomical Society of the Pacific, 122(897), 1255-1257. 